\title{
Natural Approaches for Reducing Risk Markers of Cardiovascular Diseases Related to Obesity
}

\author{
José Angel Marañón ${ }^{1 *}$, David Munoz Plaza $^{2}$ and Cristina Lozano ${ }^{3}$ \\ ${ }^{1}$ Tradichem Innovation Center, Spain \\ ${ }^{2}$ Tradichem SL, Spain \\ ${ }^{3}$ Tradichem Industrial Services, Spain
}

*Corresponding author: José Angel Marañón, Tradichem Innovation Center, Edificio CLAID, Parque Clentífico de Madrid, Campus Cantoblanco, Universidad Autónoma de Madrid, 28049, Madrid, Spain.

To Cite This Article: José Angel Marañón. Natural Approaches for Reducing Risk Markers of Cardiovascular Diseases Related to Obesity. Am J Biomed Sci \& Res. 2019 - 5(4). AJBSR.MS.ID.000937. DOI: 10.34297/AJBSR.2019.05.000937.

Received: 製September 15, 2019; Published: 帮October 01, 2019

\begin{abstract}
Raised blood lipid levels are associated with a risk of cardiovascular disease (CVD) and obesity. Despite scientific and technical progress in risk prediction, diagnostics, prognostication and therapy of cardiovascular pathologies it has been shown that nutritional intervention seems to be an effective approach to reduce the risk markers of cardiovascular diseases. Bioactive phytochemicals in food supplements are a trending approach to facilitate dieting and to improve patient's adherence to reducing food and caloric intake. This review focuses on current bioactive compounds that exhibit therapeutic effects through several cellular mechanisms in obesity and related diseases. The information discussed in the present review may provide evidence to spread the use of some effective ingredients from nature for the prevention of cardiovascular disease.
\end{abstract}

Keywords: Obesity; Cardiovascular disease; Nutrition; Phytochemicals; Cholesterol; Polysaccharides

\section{Introduction}

Calorie imbalance is related to a rising prevalence of obesity. Populations of industrialized countries are becoming more overweight as a result of changes in lifestyle, and obesity may well become the most common health problem of the 21st century. Obesity is a major contributor to the prevalence of cardiovascular disease (CVD) in the developed world, and yet has only recently been afforded the same level of attention as other risk factors of coronary artery disease (CAD). Studies indicate that higher levels of body fat are associated with an increased risk for the development of numerous adverse health conditions [1]. Weight loss is increasingly recognized to have major health benefits for overweight people [2] and also increases life expectancy in people having obesity-related complications. While reducing dietary fat content combined with increased physical exercise was shown to be effective in preventing obesity [3], only one third of those trying to lose weight reported eating fewer calories and exercising more [4]. Although weight loss and weight control drugs are becoming extremely common in today's society, the remedies provided by the diet industry have failed in the long-term maintenance of weight loss in obese patients [5]. Moreover, it has been estimated that more than $90 \%$ of the people who lose weight by dieting return to their original weight within 2-5 years [6]. Adipose tissue growth involves formation of neoadipocytes from precursor cells, further leading to an increase in adipocyte size. The transition from preadipocytes into mature adipocytes constitutes the adipocyte life cycle, and treatments that regulate both size and number of adipocytes may provide a better therapeutic approach for treating obesity. Prins and 0"Rahilly revealed for the very first time that the decrease of adipose tissue mass that occurs with weight loss may involve the mobilization of lipids through lipolysis or the loss of mature fat cells [7]. While development of obesity is a greater problem during middle age, elderly people can have a relative increase in body fat content accompanied by an accumulation of adipocytes in nonadipose tissues leading to a higher risk of stroke and other cardiovascular diseases

Bioactives from plant extracts represent the most widespread form of medication and a common tool to reduce cholesterol levels and other cardiovascular risk factors. At least $25 \%$ of the active compounds in currently prescribed synthetic drugs were first identified in plant sources [8]. Dissatisfaction with the high costs and potentially hazardous side effects of pharmaceuticals have resulted in a larger percentage of people in the United States purchasing and exploring the applications of medicinal plants than before [9]. Several plants like willow, poppy, foxglove, cinchona, aloe and garlic 
have been verified as medicinally beneficial through repeated clinical testing and laboratory analyses [10], and a number of plant extracts like green tea [11], garlic compounds [12] and conjugated linoleic acid (CLA) [13] were shown to possess either antidiabetic effects or have direct effects on adipose tissue. Substantial progresses have been made concerning our knowledge of bioactive components in plant foods and their links to obesity. Polyphenols constitute one of the ubiquitous groups of plant metabolites widely found in fruits, vegetables, cereals, legumes and wine [14]. A large number of studies have been carried out to investigate the antiobesity effects of polyphenols like apigenin and luteolin [15], kaempferol [16], myricetin and quercetin [17], genistein and daidzein [1820], cyanidin [21], grape seed proanthocyanidin extract (GSPE) [22], xanthohumol [23] and epigallocatechin gallate (EGCG) [24]. Likewise, studies involving the effects on lipid metabolism have been carried out with carotenoids like fucoxanthin [25], coumarin derivatives like esculetin [26] and phytoalexins like resveratrol [27]. Other bioactive components of food with antiobesity effects include phytosterols, polyunsaturated fatty acids and organosulfur compounds. The association between lipid and bone metabolism has become an increasing focus of interest in recent years [28], and accumulating evidence has shown that atherosclerosis and osteoporosis, a disorder of bone metabolism, frequently co-exist, therefore the reduction of CVD risk markers could have a positive effect on bone health [29].

\section{Natural compounds used for the reduction of risk biomarkers in CVD}

The use of botanicals to diminish the lipid absorption has been widely reported [30]. Plant extracts like Cissus quadrangularis [31], Aralia mandshurica (aralax) [32], psyllium [33], Salix matsudana leaves [34] and Arachis hypogaea [35] on lipid metabolism revealed a reduction in serum triglyceride levels. However, there are no major long-term studies demonstrating harm or benefit in using lipid-lowering drugs compared to low-fat diets in children [36].

Phytosterols have been widely studied for their cholesterollowering effects and because of the effect on bone health regulating the osteoblasts/osteoclast ratio [37]. Plant sterols like diosgenin, campesterol, sitosterol, stigmasterol and brassicasterol were shown to possess cholesterol lowering effects [38]. Since turnover of cholesterol was shown to bear a relationship to body fat mass [39], phytosterols may also decrease body fat. A number of studies have demonstrated the beneficial effects of polyunsaturated fatty acids (PUFAs) on lipid-related disorders in humans [40].

Adipose tissue mass can be reduced by both inhibiting adipogenesis and inducing apoptosis of adipocytes. Natural products that specifically target both these pathways therefore will have better potential for treatment and prevention of obesity. Polyphenolic compounds are widely found in fruits and vegetables among which flavonoids and several classes of nonflavonoids are usually distinguished. The antiobesity effects and also adipocyte- specific effects of several polyphenols have been investigated. PUFAs are vital components of the phospholipids of cell membranes and serve as important mediators of the nuclear events regulating the adipocyte-specific gene expression involved in lipid metabolism and adipogenesis [41,42] revealed how the Herbal composition Ob-X reduces abdominal visceral fat in humans and also shown that the visceral fat reduction reduction is linked to the decrease of the triglyceride's levels. Previously [43] suggested that a highly specific Melissa officinalis extract can reduce the adipose tissue mass by inhibiting the adipose angiogenesis. The inhibition of the angiogenesis in the adipose tissue seems related to metabolic changes in plasma lipids, liver triglycerides and weight loss.

Although most commonly used dietary supplements like CLA showed an effect on glucose and lipid metabolism, these effects are also likely secondary effects mediated through adipocyte-specific transcription factors and their nuclear receptors [44]. Likewise, although the beneficial effects of organosulfur compounds present in natural food are due to their antioxidant and anticarcinogenic properties [45], the adipocyte specific effects of ajoene, has been reported [46]. Dietary fibre (DF) primarily consists of non-starch polysaccharides, many of which are characterised by their ability to form viscous dispersions and/or gels in water [47]. For example, the consumption of $\beta$-glucan, a viscous soluble fibre from barley, can lead to a significant reduction in total cholesterol as well as LDL cholesterol levels $[48,49]$. Recent reports on the intake of barley and oat $\beta$-glucan and konjac glucomannan significantly reducing LDL cholesterol and non-HDL cholesterol levels [50-52]. Several mechanisms have been proposed to explain the beneficial effects of DF on blood lipids, including short chain fatty acid (SCFA) production in the colon, reduced fatty acid synthesis, lowered body weight with fat loss, improved glycaemic control, microbial modulation, reduced inflammation and formation of non-covalent bonds with other dietary components to adsorb or entrap them [53]. Gaining a better understanding of the beneficial influence of increased DF intake on the multiple markers for CVD risk is an important area of preventative health research.

More recently, it has been proven that water soluble polysaccharides from Agaricus Bisporus (H2Oslim $®$ ) create a cationic polimer when in contact with water, which is able to interact with anionic substances. Polimer- lipid complexes that are formed through bonds can be attributed to the density of positive charges on the polimer. [54] Because of the indigestibility of this amino polysaccharides-lipid complex, the lipid absorption by the intestine is highly reduced. These characteristics make H2Oslim ${ }^{\circledR}$ helpful in the treatment of high blood fat, hyper lipemia and high blood cholesterol, hypercholesteremia or hypertriglyceridemia. It seems that such complexes, are able to reduce the amount of lipid and cholesterol available for absorption and assimilation by the body, decreasing the most noteworthy risk markers in cardiovascular diseases in the occidental adult population. 


\section{Conclusion}

Obesity is a risk factor for diseases like non-insulin- dependent diabetes mellitus, cardiovascular diseases and certain cancers. Dietary bioactives derived from natural products have shown interesting effects on adipose tissue like inducing apoptosis, decreasing lipid accumulation and inducing lipolysis. Since a number of complex interconnected cell signaling pathways are involved in regulating all the above-mentioned processes, lipid trapping strategies and targeting the adipocytes with bioactives from ingredients could result in enhanced effects. This strategy can be achieved by exerting beneficial effects through additive or synergistic actions of several natural compounds acting at single or multiple target sites associated with physiological processes like apoptosis, adipogenesis lipolysis or inhibiting the lipid absorption. These strategies seem an effective approach in the reduction of cardiovascular risk markers.

\section{Reference}

1. Visscher TL, Seidell JC (2001) The public health impact of obesity. Annu Rev Public Health 22: 355-375.

2. Goldstein DJ (1992) Beneficial health effects of modest weight loss. Int J Obes Relat Metab Disord 16(6): 397-415.

3. Astrup A (2001) Healthy lifestyles in Europe: prevention of obesity and type II diabetes by diet and physical activity. Public Health Nutr 4(2B): 499-515.

4. Kruger J, Galuska DA, Serdula MK, Jones DA (2004) Attempting to lose weight: specific practices among U.S. adults. Am J Prevent Med 26(5): 402-426.

5. Wadden TA (1993) Treatment of obesity by moderate and severe caloric restriction. Results of clinical research trials. Ann Intern, $119(7$ Pt 2): 688-693.

6. Stern JS, Hirsch J, Blair SN, Foreyt JP, Frank A, et al. (1995) Weighing the options: criteria for evaluating weight-management programs. The Committee to Develop Criteria for Evaluating the Outcomes of Approaches to Prevent and Treat Obesity. Obes Res 3(6): 591-604.

7. Prins JB, O'Rahilly S (1997) Regulation of adipose cell number in man. Clin Sci (Lond) 92(1): 3-11

8. Balandrin MF, Klocke JA, Wurtele ES, Bollinger WH (1985) Natural plant chemicals: sources of industrial and medicinal materials. Science ;228(4704): 1154-1160.

9. Kessler RC, Davis RB, Foster DF, Van Rompay MI, Walters EE, et al. (2001) Long-term trends in the use of complementary and alternative medical therapies in the United States. Ann Intern Med 135(4): 262-268.

10. Youngkin EQ Israel DS (1996) A review and critique of common herbalalternative therapies. The Nurse practitioner; 21(10): 39, 43-46, 49-52.

11. Hasegawa N, Yamda N, Mori M (2003) Powdered green tea has antilipogenic effect on Zucker rats fed a high-fat diet. Phytother Res 17(5): 477-480.

12. Elkayam A, Mirelman D, Peleg E, Wilchek M, Miron T, et al. (2003) The effects of allicin on weight in fructose-induced hyperinsulinemic, hyperlipidemic, hypertensive rats. Am J Hypertens 16(12): 1053-1056.

13. Hargrave KM, Li C, Meyer BJ, Kachman SD, Hartzell DL, et al. (2002) Adipose depletion and apoptosis induced by trans-10, cis-12 conjugated linoleic acid in mice. Obes Res 10(12): 1284-1290.

14. Aherne SA, O'Brien NM (2002) Dietary flavonols: chemistry, food content, and metabolism. Nutrition 18(1): 75-81.

15. Han LK, Sumiyoshi M, Zheng YN, Okuda H, Kimura Y (2003) Anti-obe- sity action of Salix matsudana leaves (Part 2). Isolation of anti-obesity effectors from polyphenol fractions of Salix matsudana. Phytother Res 17(10): 1195-1198

16. Yu SF, Shun CT, Chen TM, Chen YH (2006) 3-0-beta-D-glucosyl-(1-N6)beta-D-glucosyl-kaempferol isolated from Sauropus androgenus reduces body weight gain in Wistar rats. Biol Pharm Bull 29(12): 2510-2513.

17. Kwon O, Eck P, Chen S, Corpe CP, Lee JH, et al (2007) Inhibition of the intestinal glucose transporter GLUT2 by flavonoids. FASEB J 21(2): $366-$ 377.

18. Kim HK, Nelson Dooley C, Della Fera MA, Yang JY, Zhang W, et al. (2006) Genistein decreases food intake, body weight, and fat pad weight and causes adipose tissue apoptosis in ovariectomized female mice. J Nutr 136(2): 409-414

19. Naaz A, Yellayi S, Zakroczymski MA, Bunick D, Doerge DR, et al. (2003) The soy isoflavone genistein decreases adipose deposition in mice. Endocrinology 144(8): 3315-3320.

20. Dang Z, Lowik CW (2004) The balance between concurrent activation of ERs and PPARs determines diadzein-induced osteogenesis and adipogenesis. J Bone Miner Res 19(5): 853-861.

21. Tsuda T, Ueno Y, Kojo H, Yoshikawa T, Osawa T (2005) Gene expression profile of isolated rat adipocytes treated with anthocyanins. Biochim Biophysica Acta 1733(2-3): 137-147.

22. Preuss HG, Wallerstedt D, Talpur N, Tutuncuoglu SO, Echard B, et al. (2000) Effects of niacin-bound chromium and grape seed proanthocyanidin extract on the lipid profile of hypercholesterolemic subjects: a pilot study. J Med 31(5-6): 227-246.

23. Nakagawa Y, Iinuma M, Matsuura N, Yi K, Naoi M, et al. (2008) A potent apoptosis-inducing activity of a sesquiterpene lactone, S. Rayalam, et al. (Eds.). Journal of Nutritional Biochemistry 19: 717-726 723.

24. Wolfram S, Raederstorff D, Preller M, Wang Y, Teixeira SR, et al. (2006) Epigallocatechin gallate supplementation alleviates diabetes in rodents. J Nutr 136(10): 2512-2518.

25. Maeda H, Hosokawa M, Sashima T, Funayama K, Miyashita K (2005) Fucoxanthin from edible seaweed, Undaria pinnatifida, shows antiobesity effect through UCP1 expression in white adipose tissues. Biochem Biophys Res Commun 332(2): 392-397.

26. Yang JY, Della Fera MA, Hartzell DL, Nelson Dooley C, Hausman DB, et al. (2006) Esculetin induces apoptosis and inhibits adipogenesis n 3T3-L1 cells. Obesity (Silver Spring, Md) 14(10): 1691-1699.

27. Picard F, Kurtev M, Chung N, Topark Ngarm A, Senawong T, et al. (2004) Sirt1 promotes fat mobilization in white adipocytes by repressing PPAR-gamma. Nature 429(6993): 771-776.

28. Christos Savvidis, Symeon Tournis, Anastasia D Dede (2018) Obesity and bone metabolism. Hormones 17(2): 205-217.

29. Anna Newton, Lynae Hanks, Suzanne Judd, Stephenie Wallace, Nefertiti Durant, et al. (2015) Low HDL Cholesterol May Lead to Disruption of Bone (re)Modeling in Obese Early Pubertal Girls. Int J Orthopaedics 2(3): 312-316.

30. Nan Nong Sun, Tsung Yen Wu, Chi Fai Chau (2016) Natural Dietary and Herbal Products in Anti-Obesity Treatment. Molecules. 21(10): E1351.

31. Oben JE, Enyegue DM, Fomekong GI, Soukontoua YB, Agbor GA (2007) The effect of Cissus quadrangularis (CQR-300) and a Cissus formulation (CORE) on obesity and obesity-induced oxidative stress. Lipids Health Dis 6: 4 .

32. Abidov MT, del Rio MJ, Ramazanov TZ, Klimenov AL, Dzhamirze S, et al. (2006) Effects of Aralia mandshurica and Engelhardtia chrysolepis extracts on some parameters of lipid metabolism in women with nondiabetic obesity. Bull Exp Biol Med 141(3): 343-346.

33. Moreno LA, Tresaco B, Bueno G, Fleta J, Rodriguez G, et al. (2003) Psyllium fibre and the metabolic control of obese children and adolescents. J Physiol Biochem 59(3): 235-242. 
34. Han LK, Sumiyoshi M, Zhang J, Liu MX, Zhang XF, et al. (2003) Anti-obesity action of Salix matsudana leaves (Part 1). Anti- obesity action by polyphenols of Salix matsudana in high fat-diet treated rodent animals. Phytother Res 17(10): 1188-1194.

35. Moreno DA, Ilic N, Poulev A, Raskin I (2006) Effects of Arachis hypogaea nutshell extract on lipid metabolic enzymes and obesity parameters. Life Sci 78(24): 2797-2803.

36. Bhatnagar D (2002) Should pediatric patients with hyperlipidemia receivedrug therapy? Paediatric drugs 4(4): 223-230.

37. Elke A Trautwein, Mario A Vermeer, Harry Hiemstra, Rouyanne T Ras (2018) LDL-Cholesterol Lowering of Plant Sterols and Stanols-Which Factors Influence Their Efficacy? Nutrients 10(9): 1-15.

38. Fernandez C, Suarez Y, Ferruelo AJ, Gomez Coronado D, Lasuncion MA (2002) Inhibition of cholesterol biosynthesis by Delta22-unsaturated phytosterols via competitive inhibition of sterol Delta24-reductase in mammalian cells. Biochem J 366(Pt 1): 109-119.

39. Miettinen TA (1971) Cholesterol production in obesity. Circulation 44 842-850.

40. Hirafuji M, Machida T, Hamaue N, Minami M (2003) Cardiovascular protective effects of $n-3$ polyunsaturated fatty acids with special emphasis on docosahexaenoic acid. J Pharmacol Sci 92(4): 308-316.

41. Mohsen Meydani, Syeda T Hasan (2010) Dietary polyphenols and obesity. Nutrients 2(7): 737-751.

42. J H Kang, In Sun Jeong, Min Young Kim (2018) Antiangiogenic Herbal Composition Ob-X Reduces Abdominal Visceral Fat in Humans: A Randomized, Double-Blind, Placebo-Controlled Study. Evidence-Based Complementary and Alternative Medicine (10):1-11.

43. BY Parke, Lee H, Woo S, Yoon M, Kim J, et al. (2015) Reduction of Adipose Tissue Mass by the Angiogenesis Inhibitor ALS-L1023 from Melissa officinalis. PLOS ONE 10(11): e0141612.

44. Taylor CG, Zahradka P (2004) Dietary conjugated linoleic acid and insulin sensitivity and resistance in rodent models. Am J Clin Nutr;79(6 Suppl): 1164S-1168S.
45. Sahu SC (2002) Dual role of organosulfur compounds in foods: a review. J Environ Sci Health; 20(1): 61-76.

46. Yang JY, Della Fera MA, Nelson Dooley C, Baile CA (2006) Molecular mechanisms of apoptosis induced by ajoene in 3T3-L1 adipocytes. Obes Res 14(3): 388-397.

47. Saha D, Bhattacharya S (2010) Hydrocolloids as thickening and gelling agents in food: A critical review. J Food Sci Technol 47(6): 587-597.

48. Aman P (2006) Cholesterol-lowering effects of barley dietary fibre in humans: scientific support for a generic health claim. Scand J Food Nutr 50(4):173-176

49. Schloermann W, Glei M (2017) Potential health benefits of $\beta$-glucan from barley and oat. Ernahrungs Umschau 64:145-149.

50. Ho HV, Sievenpiper JL, Zurbau A, Mejia SB, Jovanovski E, et al. (2016) The effect of oat $\beta$-glucan on LDL-cholesterol, non-HDL-cholesterol and apoB for CVD risk reduction: A systematic review and meta-analysis of randomised-controlled trials. Br J 116(8): 1369-1382.

51. Ho HVT, Sievenpiper JL, Zurbau A, Mejia SB, Jovanovski E, et al. (2016) A systematic review and meta-analysis of randomized controlled trials of the effect of barley $\beta$-glucan on LDL-C, non-HDL-C and apoB for cardiovascular disease risk reduction ${ }^{\text {i-iv }}$. Eur J Clin Nutr 70(11): 1239-1247.

52. Ho HVT, Jovanovski E, Zurbau A, Blanco Mejia S, Sievenpiper JL, et al. (2017) Systematic review and meta-analysis of randomized controlled trials of the effect of konjac glucomannan, a viscous soluble fiber, on LDL cholesterol and the new lipid targets non-HDL cholesterol and apolipoprotein B. A J Clin Nutr 105: 1239-1247.

53. Parnell JA, Raman M, Rioux KP, Reimer RA (2012) The potential role of prebiotic fibre for treatment and management of non-alcoholic fatty liver disease and associated obesity and insulin resistance. Liver Int 32(5): 701-711.

54. JA Marañón, et al. (2019) Lipid trapping capability of water-soluble polysaccharides from Agaricus bisporus. Patent application process. 